\title{
Various Care Option of Integrative Medicine from the Viewpoint of Patient-Oriented Medicine
}

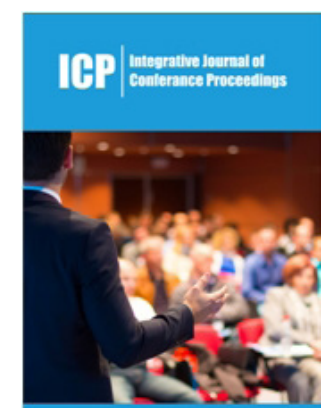

*Corresponding author: Bando H, Medical Research/Tokushima University Tokushima, Japan

Submission: 佂 December 20, 2019

Published: 㘹January 10, 2020

Volume 2 - Issue 1

How to cite this article: Bando H, Yoshioka A, Nishikiori Y. Various Care Option of Integrative Medicine from the Viewpoint of Patient-Oriented Medicine. Int J Conf Proc.2(1). ICP.000529.2020.

Copyright@ Bando H, This article is distributed under the terms of the Creative Commons Attribution 4.0 International License, which permits unrestricted use and redistribution provided that the original author and source are credited.

\author{
Bando $\mathrm{H}^{1,2 *}$, Yoshioka $\mathrm{A}^{2}$ and Nishikiori $\mathrm{Y}^{2}$ \\ ${ }^{1}$ Tokushima University, Japan \\ ${ }^{2}$ Shikoku Division, Integrative Medicine Japan, Japan
}

\begin{abstract}
Authors and colleagues have managed Shikoku Island division of Integrative Medicine Japan (IMJ) and continued various activities. There were several important conferences in 2019 concerning Integrative Medicine (IM) and Complementary and Alternative Medicine (CAM). World Federation of Chinese Medicine Societies held the $4^{\text {th }}$ cancer rehabilitation seminar in Nagoya, as cooperation with IMJ in May. There was annual congress of our Shikoku division in October, with 14 various lectures such as music therapy, chiropractic, rehabilitation, Hinohara-ism and so on. Annual IMJ congress was held at Kagoshima in December with 1000 attendees and 100 presentations. The keynote speech by Dr. Noriko Yoshida was impressive for remarkable projects, such as national health project, long life \& treasure child project, music exercise therapy with traditional song and dance, centenarian research project, island health thalasso-tourism, and psychotherapy. Author gave comments about mindfulness originated from Budhism, Pythagoreanism from Pythagoras and Hinohara-ism from famous Japanese physician Shigeaki Hinohara. These philosophies have common aspect of patient-oriented medicine, which are crucial element for IM and CAM.
\end{abstract}

Keywords: Integrative Medicine Japan (IMJ); Hippocratic oath; Hinohara-ism; Patient-oriented medicine; Pythagoras; Mindfulness

Abbreviation: IM: Integrative Medicine; CAM: Complementary and Alternative Medicine; IMJ: Integrative Medicine Japan

\section{Commentary}

In recent years, Integrative Medicine (IM) and Complementary and Alternative Medicine (CAM) have been developing in many countries and districts across the world. In European and North American countries, there have been a variety of activities and medical journals concerning IM and CAM, such as Integrative Journal of Conference Proceedings [1]. On the other hand, we have continued various activities for IM and CAM in Japan as Integrative Medicine Japan (IMJ) [2]. Furthermore, author and colleagues have managed Shikoku Island division of IMJ and reported some studies [3]. From some consecutive activities of IMJ, some developments during 2019 would be introduced in this article. The significance and perspective of IM and CAM for the future would be also described. As one of the collaborative congress of IMJ, World Federation of Chinese Medicine Societies held the $4^{\text {th }}$ cancer rehabilitation seminar in Nagoya, Japan in May 2019 [4]. IMJ president Prof. Ito and executive chairman Prof. Fukuzawa had main role of managing 20 plenary lectures. The themes included Chinese medicine, cancer rehabilitation, integrative oncology, psychological approach, aromatherapy, sociology, nutrition, as well as my lecture concerning music therapy / low carbohydrate diet (LCD). The theme of this international congress was Co-fusion and sharing to build health of cancer patients [4]. The venue was Aichi Medical University, where there is the stone of

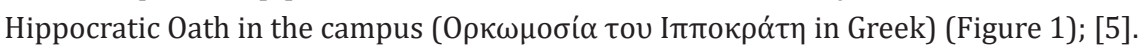

Japan consists of four main islands, which are Hokkaido, Honshu, Shikoku and Kyushu. We held $12^{\text {th }}$ annual congress of Shikoku division of IMJ in October 2019. There were 14 various lectures and presentations including music therapy, nutrition, cancer therapy, chiropractic, yoga, Chinese medicine, rehabilitation, psychotherapy, radiology, Hinohara-ism, and so 
on. After successful congress, Shikoku bulletin of IM No.12 was summarized with 40 authors and 100 pages [6]. This is only annual bulletin published in the division of IMJ, which has ISSN number. The characteristic strong point of this bulletin would be the influence of developing IM and CAM for various occupational staffs in the actual medical practice. Currently, it has been Japanese language version, and we are also planning to develop internet version with English language. Many co-medical staffs have attended and presented their papers for years. Consequently, we expect further development and collaboration in the field of IM and CAM.

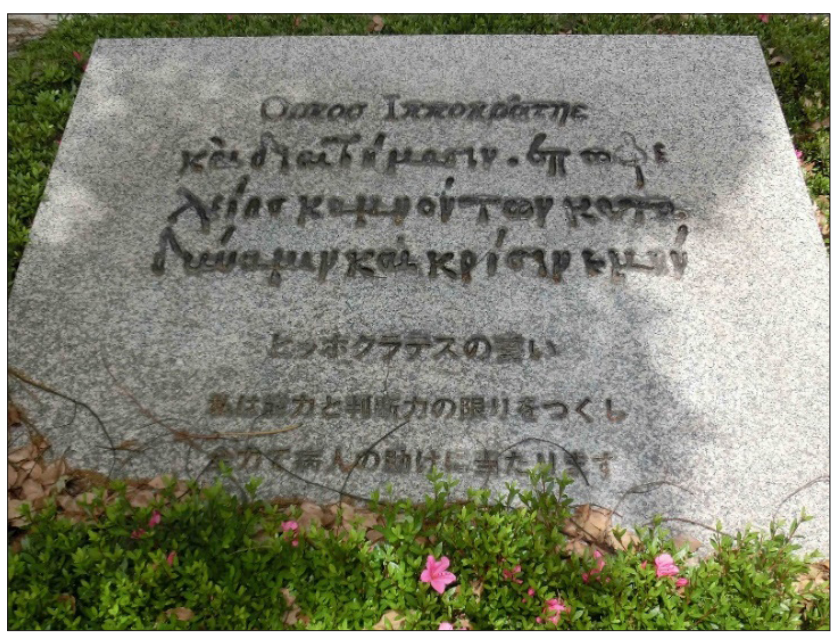

Figure 1:

There was $23^{\text {rd }}$ annual congress of IMJ at Kagoshima in December 2019 [7]. We had more than 1000 attendees, 39 lectures/symposia/ workshops and $60 \mathrm{oral} /$ poster presentations. The chairman was Dr. Noriko Yoshida and her keynote speech was impressive and excellent. She has continued developing medical and health care systems in Kagoshima more than 30 years in cooperation with public hospitals and local government. There have been lots of remarkable projects such as national health project, long life \& treasure child project, music exercise therapy with traditional song and dance, centenarian research project, prevention of flail and locomotive syndrome, thalassotherapy, island health thalasso-tourism, medical village tourism, international communication and development, and so on. In this congress, the author served a chair in the section of psychological and psychosomatic medicine. Recently, there have been a variety of discussion concerning mindfulness in the light of medical, cultural and social aspects [8]. The word "mindfulness" has the origin from Buddhism, which was Samma-Sati meaning right thinking [9]. Taking the advantages of this concept, people can keep their mind, heart and spirit rather calm with stable condition. Consequently, adequate lecture or advice about mindfulness would be helpful for patients with various problems.

The author has been a physician, pianist, and registered music therapist. The concept of mindfulness is related to the principle of music therapy. Formerly in Inoian Greek era, there was a famous philosopher, Pythagoras (BC 582-496, who is described as Пu$\theta \alpha \gamma o ́ p \alpha \varsigma$ (Greek) and Pȳthagórās (Latin) [10]. He was the founder of Pythagoreanism and was also a musician and discovered the basis of music scale with 12 notes, ie., Do Re Mi (Figure 2). He recommended people to live every day with harmonized condition of heart and mind. Even if an accident occurs, keeping the sympathy stable would bring the healthy life.

Furthermore, he described as follows:

a. Beautiful music is flowing in the heavenly universe.

b. Ordinary people can't hear the music, but only a few people with clean and transparent mind can listen to it.

c. These ideas seem to be based on oriental way of thinking and culture, which can become a meaningful reference to western culture.

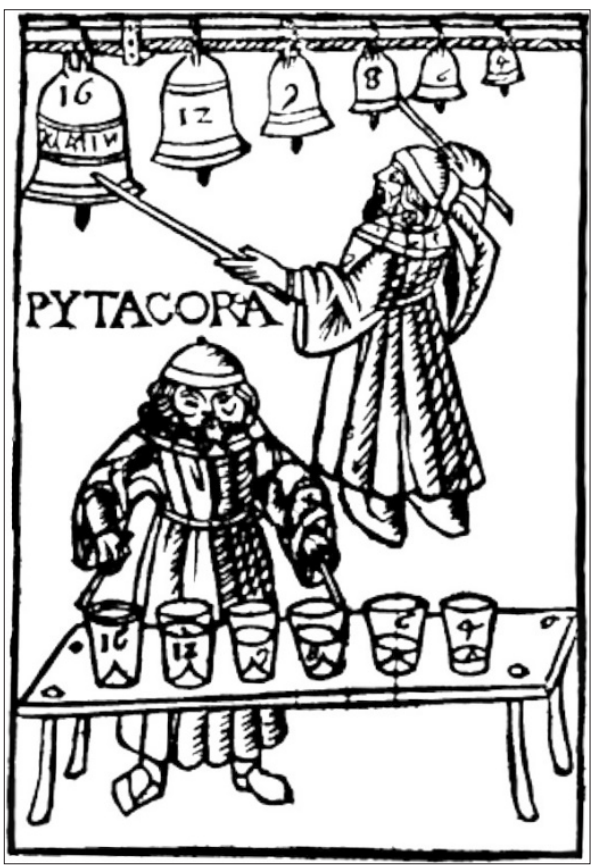

Figure 2:

There were excellent physicians who were also philosopher and teacher in the medical history. In North American region, Sir William Osler has been well-known, who was a Professor of Johns Hopkins University [11]. He said as follows: "Listen to your patient; he is telling you the diagnosis". His philosophy has been passed down to the younger generation doctors as the Oslerism [12]. The great achievement of Dr. Osler was introduced by Shigeaki Hinohara, who was a prominent Japanese physician living up to 105 years [13]. He was also an excellent pioneer for developing medical education, as well as musician and philosopher. Dr. Hinohara described that medicine, religion and music have the same origin from the viewpoint of human evolution. Hinohara-ism has been widely accepted and been more prevalent in medical education, nursing, primary care, practice of medicine for long years [14]. Sir Osler [11] and Dr. Hinohara [13] had emphasized the importance of patient-oriented medicine $[15,16]$. The author has several opportunities for enlightenment of IM and CAM.

In such case, the similarity of the medical specialty would be discussed including IM, CAM, primary care medicine, family 
medicine, psychosomatic medicine. Their common viewpoint would be the integrated holistic care physically and psychologically. It is like not ready-made clothes but order-made clothes (pret-aporter). The meaning of the health is originally derived from the word "Swasth" in Sanskrit language [17]. Swasth is made of SWA (my, inner self) +STH (exist, keep, conscious), which means "selfexistence under self-consciousness" [18]. On the other hand, outer self-consciousness with several matters of body role which are everchanging, and mortal can lead to insecurity and instability [19]. In modern era worldwide, health in each person can be maintained by various kinds of therapy. From broad defense ability and power IM can respond to a variety of health problems for adequate and satisfactory care and cure at present and also in the future.

\section{References}

1. Nakanishi A, Bando H (2019) Development of health resort casino system in integrative medicine. Int J Conf Proc 1(5): 1-3.

2. http://imj.or.jp/

3. Yoshioka A, Bando H, Nishikiori Y, Nakanishi A (2019) Recent status of hydrotherapy and balneotherapy with clinical beneficial effects. Int J Complement Alt Med 12(6): 217-219.

4. http://imj.or.jp/new/wp/wp-content/uploads/seminar_20190518.pdf

5. Weeks J (2017) A hippocratic oath for integrative health practitioners. Alternative Medicine, Minnesota, USA, pp. 18-19.

6. Bando H (2019) Bulletin of shikoku division of integrative medicine japan (IMJ) Shikoku, Japan 12: 1-100.

7. http://imj2019.com/

8. Kemper KJ, Carmin C, Mehta B, Binkley P (2016) Integrative medical care plus mindfulness training for patients with congestive heart failure. J Evid Based Complementary Altern Med 21(4): 282-290.

9. Nathan N (2017) Being mindful in managing pain: Integrative medicine in chronic pain management. Anesth Analg 125 (6): 1828.

10. Bando H (2019) Music therapy (eBook). Academic Publishing, Adana, Turkey, pp. 186-246.

11. Osler W (1904) On the educational value of the medical society. In Aequanimitas with other addresses to medical students, nurses and practitioners of medicine, Philadelphia, USA, pp. 343-362.

12. Seeman MV, Becker RE (2017) Osler and the way we were taught. Med Sci Educ 27(3): 555-557.

13. Hinohara S (1993) Sir william osler's philosophy on death. Annals of Internal Medicine 118(8): 638-642.

14. Bando H, Yoshioka A, Iwashimizu Y, Iwashita M, Doba N (2017) Development of primary care, lifestyle disease and new elderly association (Nea) in japan-common philosophy with hinohara-ism. Prim Health Care 7(3): 1-5.

15. Stafford N (2017) Shigeaki hinohara. BMJ 358: j3891.

16. Bando H (2018) Philosophy of internal medicine-Osler and hinoharaism. Intern Med Open J 2(1): 7-9.

17. Khade SC, Gharge A (2018) Innermost reconciliation through vipassana. Ayurlog: National Journal of Research in Ayurved Science 6(1): 1-6.

18. Kumar K (2018) Human mind, mental processes and role of yoga in mental health. International Journal of Science and Consciousness 4(2): 48-54.

19. Gupta SK, Sawhney RC, Rai L, Chavan VD, Dani S, et al. (2011) Regression of coronary atherosclerosis through healthy lifestyle in coronary artery disease patients-Mount Abu open heart trial. Indian Heart J 63(5): 461469.

For possible submissions Click below: 